\title{
The effect of cost on adherence to prescription medications in Canada
}

\author{
Michael R. Law PhD, Lucy Cheng MSc, Irfan A. Dhalla MD MSc, Deborah Heard BASc, Steven G. Morgan PhD
}

\begin{abstract}
Background: Many patients do not adhere to treatment because they cannot afford their prescription medications, putting them at increased risk of adverse health outcomes. We determined the prevalence of cost-related nonadherence and investigated its associated characteristics, including whether a person has drug insurance.
\end{abstract}

Methods: Using data from the 2007 Canada Community Health Survey, we analyzed the responses of 5732 people who answered questions about cost-related nonadherence to treatment. We determined the national prevalence of cost-related nonadherence and used logistic regression to evaluate the association between cost-related nonadherence and a series of demographic and socioeconomic variables, including province of residence, age, sex, household income, health status and having drug insurance.

Results: Cost-related nonadherence was reported by $9.6 \%$ ( $95 \%$ confidence interval
[CI] $8.5 \%-10.6 \%$ ) of Canadians who had received a prescription in the past year. In our adjusted model, we found that people in poor health (odds ratio [OR] 2.64, 95\% Cl 1.77-3.94), those with lower income (OR 3.29, 95\% Cl 2.03-5.33), those without drug insurance (OR 4.52, 95\% Cl 3.29-6.20) and those who live in British Columbia (OR 2.56, $95 \% \mathrm{Cl} 1.49-4.42$ ) were more likely to report cost-related nonadherence. Predicted rates of cost-related nonadherence ranged from $3.6 \%$ (95\% Cl 2.4-4.5) among people with insurance and high household incomes to $35.6 \%$ (95\% Cl 26.1\%-44.9\%) among people with no insurance and low household incomes.

Interpretation: About 1 in 10 Canadians who receive a prescription report costrelated nonadherence. The variability in insurance coverage for prescription medications appears to be a key reason behind this phenomenon.
Competing interests: Michael Law has consulted for Health Canada on a related report on costrelated nonadherence to prescription drugs. Irfan Dhalla is a volunteer member of the board of Canadian Doctors for Medicare and a paid member of the Committee to Evaluate Drugs in Ontario. Steven Morgan has been a consultant to federal and provincial governments on matters related to pharmaceutical policy. No other competings interests were declared.

This article has been peer reviewed.

Correspondence to: Dr. Michael R. Law, mlaw@chspr.ubc.ca

CMAJ 2012. DOI:10.1503 /cmaj.111270
$\mathrm{O}$ ver half of the total spending on prescription drugs in Canada is for medications intended for long-term use, such as drugs to manage cardiovascular risk factors. ${ }^{1,2}$ Suboptimal adherence to prescription medications is a well-known impediment to effective treatment and, consequently, an impediment to better health outcomes. ${ }^{3} \mathrm{Al}$ though several factors may influence suboptimal adherence to medication, previous research suggests that cost may be the most important factor amenable to policy intervention. ${ }^{4,5}$

Outpatient prescription medications fall outside the scope of the Canada Health Act, which requires provinces to provide universal public insurance for medically necessary hospital and physician services. Prescription drug financing in Canada is therefore a "patchwork" that includes out-of-pocket payments that depend on whether and to what extent one has access to a private or public insurance plan. ${ }^{6}$ As a result, two-thirds of Canadian households incur out-of-pocket expenses for prescription drugs each year. ${ }^{7}$ These payments totaled $\$ 4.6$ billion in 2010 , or about $17.5 \%$ of total spending on prescription drugs. ${ }^{8}$ One concern over these substantial out-of-pocket contributions is patients not adhering to necessary medications (i.e., they may not fill prescriptions, or they might skip doses).

Previous research, mostly from the United States, has shown that such cost-related nonadherence to treatment is widespread and related to not having insurance coverage for prescription drugs. ${ }^{9-11}$ However, little is known about this phenomenon in Canada. ${ }^{12}$ In addition, the most recent estimate regarding the prevalence of costrelated nonadherence in Canada was based on a survey with a response rate of $29 \% .^{13}$ 
We used data from a large national survey to determine the prevalence of cost-related nonadherence and its associated individual characteristics, including whether a person has insurance coverage for prescription drugs.

\section{Methods}

\section{Data and variables}

We analyzed data from the 2007 Canadian Community Health Survey, a telephone survey of the community-dwelling household population 12 years of age and older. ${ }^{14}$ From September to December, 2007, respondents were asked a series of questions on prescription drug insurance and cost-related nonadherence. The response rate to this component of the survey was $72.2 \%$ and included 10898 respondents from all 10 provinces. Of these respondents, 9404 $(86.3 \%)$ agreed to share their data with Statistics Canada's partner agencies. Our study sample was drawn from these respondents.

Cost-related nonadherence was measured using a combination of responses to three questions on the survey (questions 3-5, Appendix 1, available at www.cmaj.ca/lookup/suppl/doi:10 $.1503 / \mathrm{cmaj} .111270 /$-/DC1). Respondents were first asked whether they had received a prescription in the past year. Those who reported that they had received a prescription were then asked three questions about whether costs led them to do anything to make their prescription last longer, not fill a new prescription or not renew a prescription. If a respondent answered yes to any of these three questions, we considered this to be a report of cost-related nonadherence.

We used the survey to construct a range of variables that are consistent with widely used conceptual frameworks on access to health services. ${ }^{15}$ These included province of residence, age, sex, household income, level of education, self-assessed health status and number of reported chronic conditions (including arthritis, chronic obstructive pulmonary disease, diabetes, cancer, heart disease, high blood pressure and mood disorders), as well as whether respondents reported having prescription drug coverage that paid all or part of the cost of their medications (question 1, Appendix 1).

\section{Statistical analysis}

We developed a logistic regression model to determine what factors were associated with reporting cost-related nonadherence. Because the number of respondents in some provinces was small, we combined provinces with less than 1000 respondents into two regional groups: Saskatchewan and Manitoba were combined, as were New Brunswick, Nova Scotia, Prince Edward Island, and Newfoundland and Labrador (Atlantic provinces). This grouping did not change our results by individual province in any substantive manner. We grouped several other variables into categories, including age (12-34, $35-44,45-64$, and $\geq 65$ years), annual household income $(<\$ 20000, \$ 20000-\$ 39999, \$ 40$ 000-\$59 999, \$60 000-\$79 999, $\geq \$ 80000$ ), highest level of education attained (less than high school, high school, some postsecondary, postsecondary), self-assessed health status (excellent or very good, good, fair or poor), and number of reported chronic conditions (none, one, two or more).

To account for the complex sampling design of the survey, we used Statistics Canada survey weights and calculated all confidence intervals (CIs) using bootstrapping. ${ }^{16}$ In our descriptive analysis, we included only respondents for whom we had complete data $(n=7917,84.2 \%)$. When calculating rates of cost-related nonadherence and statistical modelling, we further restricted our sample to those who reported having received a prescription in the past year $(n=$ $5732,72.4 \%$ ). We also conducted two sensitivity analyses. First, we included respondents for whom income data was missing in our model and used multiple imputation based on chained equations to estimate their incomes. ${ }^{17}$ Second, we modelled an outcome variable calculated from only the first two cost-related questions on nonadherence (not filling and not renewing a prescription). Both analyses showed results with the same substantive interpretation as those we report here (data not shown). To facilitate interpretation of our regression model, we predicted the probability of cost-related nonadherence for both insured and uninsured members of each income group. We calculated these predictions using the average for all other covariates to make them representative of the entire Canadian population.

\section{Results}

After weighting the number of respondents who reported cost-related nonadherence to represent the overall Canadian population, we found that $9.6 \%$ (95\% CI $8.4 \%-10.7 \%$ ) of people who received a prescription reported engaging in one or more forms of cost-related nonadherence in the year preceding the survey (Table 1). At the provincial level, rates of cost-related nonadherence were lowest in Quebec $(7.2 \%, 95 \% \mathrm{CI}$ 4.5\%-9.8\%) and highest in British Columbia $(17.0 \%, 95 \%$ CI $12.6 \%-21.4 \%)$. Cost-related nonadherence was most common within the pop- 
Table 1: Characteristics and prevalence of cost-related nonadherence among respondents to the Canadian Community Health Survey in 2007

\begin{tabular}{|c|c|c|c|c|}
\hline Characteristic & $\begin{array}{c}\text { Respondents with } \\
\text { complete data, } \\
\text { no. }\end{array}$ & $\begin{array}{c}\text { Respondents } \\
\text { who received } \\
\text { a prescription, } \\
\text { no. }\end{array}$ & $\begin{array}{l}\text { Respondents who } \\
\text { reported cost- } \\
\text { related } \\
\text { nonadherence, } \\
\text { no. }\end{array}$ & $\begin{array}{c}\text { Weighted prevalence } \\
\text { of cost-related } \\
\text { nonadherence, }{ }^{*} \\
\%(95 \% \mathrm{Cl})\end{array}$ \\
\hline Overall & 7917 & 5732 & 540 & $9.6 \quad(8.4-10.7)$ \\
\hline \multicolumn{5}{|l|}{ Province } \\
\hline Atlantic provinces & 1191 & 889 & 105 & $11.9 \quad(8.9-14.9)$ \\
\hline Quebec & 1542 & 1062 & 68 & $7.2 \quad(4.5-9.8)$ \\
\hline Ontario & 2629 & 1932 & 168 & $9.1 \quad(7.2-11.0)$ \\
\hline $\begin{array}{l}\text { Saskatchewan and } \\
\text { Manitoba }\end{array}$ & 947 & 686 & 71 & $8.9 \quad(6.0-11.7)$ \\
\hline Alberta & 777 & 565 & 40 & $7.6 \quad(4.6-10.7)$ \\
\hline British Columbia & 831 & 598 & 88 & $17.0(12.6-21.4)$ \\
\hline \multicolumn{5}{|l|}{ Age, yr } \\
\hline $12-34$ & 2116 & 1295 & 135 & $9.4 \quad(7.2-11.6)$ \\
\hline $35-44$ & 1253 & 809 & 86 & $11.4 \quad(8.2-14.7)$ \\
\hline $45-64$ & 2813 & 2121 & 229 & $10.8 \quad(8.7-12.9)$ \\
\hline$\geq 65$ & 1735 & 1507 & 90 & $4.8 \quad(3.6-6.0)$ \\
\hline \multicolumn{5}{|l|}{ Sex } \\
\hline Female & 4363 & 3453 & 358 & $10.7 \quad(9.2-12.2)$ \\
\hline Male & 3554 & 2279 & 182 & $8.2 \quad(6.4-9.9)$ \\
\hline \multicolumn{5}{|l|}{ Health status } \\
\hline Excellent or very good & 4573 & 2993 & 207 & $6.9 \quad(5.6-8.2)$ \\
\hline Good & 2341 & 1820 & 173 & $10.4 \quad(8.0-12.8)$ \\
\hline Fair or poor & 1003 & 919 & 160 & $20.1(15.7-24.4)$ \\
\hline \multicolumn{5}{|l|}{ Chronic conditions, no. } \\
\hline 0 & 4687 & 2797 & 221 & $8.2 \quad(6.7-9.7)$ \\
\hline 1 & 1880 & 1637 & 171 & $11.2 \quad(8.7-13.7)$ \\
\hline$\geq 2$ & 1350 & 1298 & 148 & $12.4 \quad(9.8-15.1)$ \\
\hline \multicolumn{5}{|l|}{$\begin{array}{l}\text { Annual household } \\
\text { income, \$ }\end{array}$} \\
\hline$<20000$ & 948 & 764 & 136 & $20.5(15.2-25.8)$ \\
\hline $20000-39999$ & 1663 & 1270 & 143 & $13.7(10.7-16.7)$ \\
\hline $40000-59999$ & 1469 & 1054 & 102 & $10.5 \quad(7.8-13.3)$ \\
\hline $60000-79999$ & 1236 & 867 & 71 & $10.4 \quad(7.1-13.6)$ \\
\hline$\geq 80000$ & 2601 & 1777 & 88 & $5.4 \quad(3.9-6.9)$ \\
\hline \multicolumn{5}{|l|}{ Level of education } \\
\hline Less than high school & 1787 & 1228 & 100 & $7.3 \quad(4.9-9.8)$ \\
\hline High school & 1213 & 861 & 81 & $11.9 \quad(8.1-15.6)$ \\
\hline Some postsecondary & 516 & 376 & 48 & $12.8 \quad(7.8-17.7)$ \\
\hline Postsecondary graduate & 4401 & 3267 & 311 & $9.2(7.8-10.6)$ \\
\hline \multicolumn{5}{|l|}{$\begin{array}{l}\text { Insurance coverage for } \\
\text { prescription drugs }\end{array}$} \\
\hline Yes & 6506 & 4840 & 337 & $6.8 \quad(5.7-7.8)$ \\
\hline No & 1411 & 892 & 203 & $26.5(21.8-31.3)$ \\
\hline
\end{tabular}


ulation of people under the age of 65 years, peaking among respondents aged 35-44 years (11.4\%, 95\% CI 8.2\%-14.7\%). Unadjusted rates of cost-related nonadherence were higher among people with lower household incomes and people with poorer health. Cost-related nonadher-

\begin{tabular}{|c|c|}
\hline Variable & OR $(95 \% \mathrm{Cl})$ \\
\hline \multicolumn{2}{|l|}{ Province } \\
\hline Atlantic provinces & $1.21(0.71-2.07)$ \\
\hline Quebec & $1.26(0.76-2.07)$ \\
\hline Ontario (reference) & 1.00 \\
\hline Saskatchewan and Manitoba & $1.08(0.59-1.97)$ \\
\hline Alberta & $1.19(0.61-2.31)$ \\
\hline British Columbia & $2.56(1.49-4.42)$ \\
\hline \multicolumn{2}{|l|}{ Age, yr } \\
\hline $12-34$ & $4.70(2.91-7.60)$ \\
\hline $35-44$ & $5.57(3.45-8.98)$ \\
\hline $45-64$ & $3.74(2.55-5.49)$ \\
\hline$\geq 65$ (reference) & 1.00 \\
\hline \multicolumn{2}{|l|}{ Sex } \\
\hline Female & $1.17(0.86-1.59)$ \\
\hline Male (reference) & 1.00 \\
\hline \multicolumn{2}{|l|}{ Health status } \\
\hline Excellent or very good (reference) & 1.00 \\
\hline Good & $1.35(0.95-1.93)$ \\
\hline Fair or poor & $2.64(1.77-3.94)$ \\
\hline \multicolumn{2}{|l|}{ Chronic conditions } \\
\hline 0 (reference) & 1.00 \\
\hline 1 & $1.35(0.92-1.98)$ \\
\hline$\geq 2$ & $1.61(1.07-2.43)$ \\
\hline \multicolumn{2}{|l|}{ Annual household income, \$ } \\
\hline$<20000$ & $3.29(2.03-5.33)$ \\
\hline 20 000-39999 & $2.47(1.59-3.84)$ \\
\hline $40000-59999$ & $1.84(1.18-2.85)$ \\
\hline $60000-79999$ & $1.87(1.17-3.00)$ \\
\hline$\geq 80000$ (reference) & 1.00 \\
\hline \multicolumn{2}{|l|}{ Level of education } \\
\hline Less than high school & $0.58(0.33-1.04)$ \\
\hline High school (reference) & 1.00 \\
\hline Some postsecondary & $1.12(0.61-2.05)$ \\
\hline Postsecondary graduate & $0.99(0.65-1.50)$ \\
\hline \multicolumn{2}{|l|}{$\begin{array}{l}\text { Insurance coverage for } \\
\text { prescription drugs }\end{array}$} \\
\hline Yes (reference) & 1.00 \\
\hline No & $4.52(3.29-6.20)$ \\
\hline
\end{tabular}

ence was reported by $26.5 \%$ of respondents who had no drug coverage (95\% CI 21.8\%-31.3\%); of the people who did have insurance coverage for prescription drugs, only $6.8 \%$ (95\% CI $5.7 \%-7.8 \%$ ) reported cost-related nonadherence.

After multivariate adjustment, we found that lacking insurance for prescription drugs was associated with a more than fourfold increase in the odds of cost-related nonadherence (odds ratio [OR] 4.52, 95\% CI 3.29-6.20) (Table 2). Similarly, low household income was associated with an increase in cost-related nonadherence relative to the high household income (OR 3.29, 95\% CI 2.03-5.33) (Table 2). Cost-related nonadherence was also concentrated among respondents with the lowest self-assessed health status. People reporting fair or poor health status were more than twice as likely (OR 2.64, 95\% CI 1.773.94) to report cost-related nonadherence than respondents reporting excellent or very good health (Table 2). Similarly, respondents reporting two or more chronic conditions were 1.61 times more likely (95\% CI 1.07-2.43) to report costrelated nonadherence than respondents who reported none (Table 2). Other factors associated with higher rates of cost-related nonadherence included living in British Columbia and being younger than 65 years of age (Table 2).

The predicted probabilities of cost-related nonadherence by level of income, for both insured and noninsured respondents, are shown in Figure 1. Overall, the predicted probabilities of reporting cost-related nonadherence range from $3.6 \%$ (95\% CI 2.5-4.5) among people with insurance and high household incomes to $35.6 \%$ among people with low household incomes and no insurance (95\% CI 26.1-44.9).

\section{Interpretation}

We found that nearly 1 in 10 Canadians who received a prescription reported that out-ofpocket expenses led them to not fill a prescription, not renew a prescription, or try to make an existing prescription last longer. Furthermore, we found that several patient characteristics are associated with cost-related nonadherence: not having insurance coverage for prescription drugs, being in poor health, having a low household income, being under the age of 65 years and living in British Columbia.

Our results are consistent with those from previous studies in the US. For example, one review found that not having insurance was associated with higher cost-related nonadherence in 17 different studies. ${ }^{10}$ Our study provides evidence that gaps in drug coverage may play a similar role in Canada. Furthermore, American 


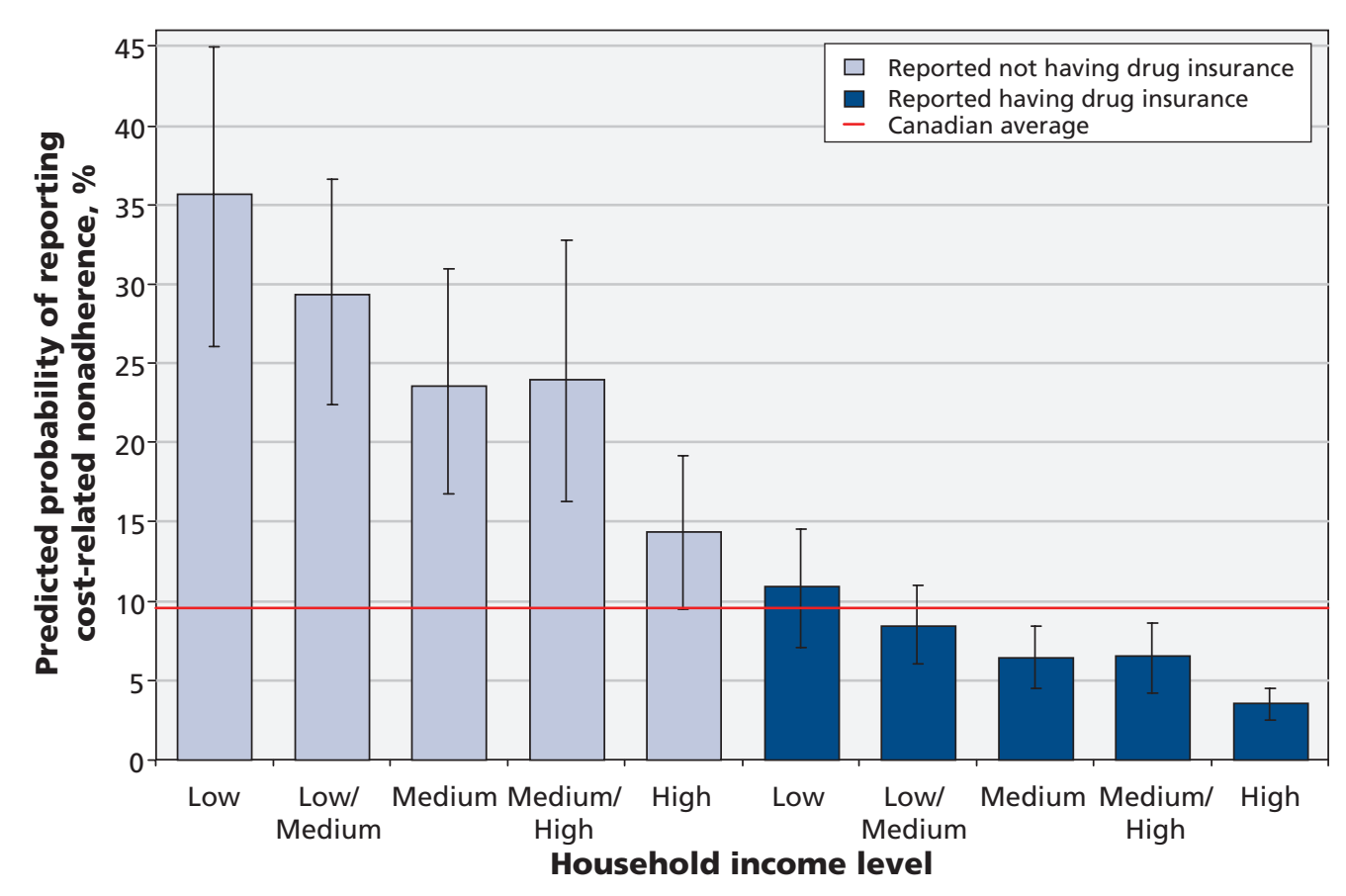

Figure 1: Predicted probabilities of cost-related nonadherence among Canadians by income level. Error bars indicate $95 \%$ confidence intervals. Income levels are based on annual household incomess: low = less than $\$ 20000$, low/medium $=\$ 20000-\$ 39999$, medium $=\$ 40000-\$ 59999$, medium $/$ high $=\$ 60000-\$ 79999$, high $=\$ 80000$ or more.

studies have consistently found that having a lower income, being in poorer health and being a younger adult are associated with higher rates of cost-related nonadherence..$^{10}$ Overall, our result that $9.6 \%$ of Canadians who have received a prescription engage in cost-related nonadherence is reasonably consistent with previous estimates and is lower than comparable estimates from the US. ${ }^{12,18}$

We did not expect that cost-related nonadherence would be highest in British Columbia. This finding might result from that province's highdeductible public drug plan or the high level of personal debt among its residents. ${ }^{19}$

\section{Limitations}

As with any study of this nature, it is possible that the data we obtained through self-report is inaccurate. In addition, we could not differentiate between private and public insurance plans or identify people who may have incorrectly reported not having coverage through a public plan..$^{20}$

We lacked a large enough sample for subgroup analysis on populations such as people with specific chronic conditions or mental illnesses. We also lacked information on the specific prescription medications that were avoided due to their cost, thus limiting our ability to make inferences as to the consequences of costrelated nonadherence.

\section{Conclusion}

For many years, there has been increasing concern over the growing financial burden imposed on Canadians by out-of-pocket expenses incurred for prescription drugs. ${ }^{21}$ Our results suggest that these costs lead many Canadians to not adhere to their prescription medications, particularly people with low incomes, people with illnesses or people who do not have insurance. Reducing cost-related nonadherence would likely improve health and reduce spending in other areas, such as admissions to hospital for acute care..$^{22}$ Of all the factors we found to be associated with cost-related nonadherence, insurance coverage is the most amenable to being addressed through changes in public policy. ${ }^{5}$

\section{References}

1. Morgan S, Raymond C, Mooney D, et al. The Canadian Rx atlas. Vancouver (BC): Centre for Health Services and Policy Research, University of British Columbia; 2008.

2. Canadian Institute for Health Information. Health care in Canada, 2008. Ottawa (ON): The Institute; 2008.

3. Osterberg L, Blaschke T. Adherence to medication. $N$ Engl J Med 2005;353:487-97.

4. Lexchin J, Grootendorst P. Effects of prescription drug user fees on drug and health services use and on health status in vulnerable populations: a systematic review of the evidence. Int $J$ Health Serv 2004;34:101-22.

5. Goldman DP, Joyce GF, Zheng Y. Prescription drug cost sharing: associations with medication and medical utilization and spending and health. JAMA 2007;298:61-9.

6. Kapur V, Basu K. Drug coverage in Canada: Who is at risk? Health Policy 2005;71:181-93.

7. CANSIM Table 109-5012. Ottawa (ON): Statistics Canada; 2009. 
Available: www5.statcan.gc.ca/cansim/pick-choisir?lang=eng \&searchTypeByValue=1\&id=1095012 (accessed 2011 Mar. 5).

8. Canadian Institute for Health Information. Drug expenditure in Canada, 1985 to 2010. Ottawa (ON): The Institute; 2010.

9. Soumerai SB, Pierre-Jacques M, Zhang F, et al. Cost-related medication nonadherence among elderly and disabled medicare beneficiaries: a national survey 1 year before the Medicare drug benefit. Arch Intern Med 2006;166:1829-35.

10. Briesacher BA, Gurwitz JH, Soumerai SB. Patients at-risk for cost-related medication nonadherence: a review of the literature. J Gen Intern Med 2007;22:864-71.

11. Madden JM, Graves AJ, Zhang F, et al. Cost-related medication nonadherence and spending on basic needs following implementation of medicare, Part D. JAMA 2008;299:1922-8.

12. Kennedy J, Morgan S. A cross-national study of prescription nonadherence due to cost: data from the joint Canada-United States Survey of Health. Clin Ther 2006;28:1217-24.

13. Schoen C, Osborn R, Squires D, et al. How health insurance design affects access to care and costs, by income, in eleven countries. Health Aff (Millwood) 2010;29:2323-34.

14. Canadian Community Health Survey (CCHS) - Annual component. User guide. Ottawa (ON): Statistics Canada; 2009. Available: www.statcan.gc.ca/imdb-bmdi/document/3226_D7_T9_V6 -eng.pdf (accessed 2011 July 27).

15. Andersen RM. Revisiting the behavioral model and access to medical care: Does it matter? J Health Soc Behav 1995;36:1-10.

16. Bootvar User Guide (Version 3.2 - SAS). Ottawa (ON): Statistics Canada; 2010.

17. Horton NJ, Lipsitz SR. Multiple imputation in practice. Am Stat 2001;55:244-54

18. Schoen C, Osborn R, Doty MM, et al. Toward higher-performance health systems: adults' health care experiences in seven countries, 2007. Health Aff (Millwood) 2007;26:w717-34.

19. Alexander C, Burleton D, Petramala D. Assessing the financial vulnerability of households across Canadian regions. Toronto $(\mathrm{ON})$ : TD Economics; 2011. Available: www.td.com/document/PDF/economics /special/td-economics-special-db0211-householddebt.pdf (accessed 2011 Dec. 9)

20. Grootendorst P, Newman EC, Levine MAH. Validity of selfreported prescription drug insurance coverage. Health Rep 2003; 14:35-46.

21. Stanbrook MB, Hébert PC, Coutts J, et al. Can Canada get on with national pharmacare already? CMAJ 2011;183:E1275.

22. Dhalla I, Smith M, Choudhry N, et al. Costs and benefits of free medications after myocardial infarction. Healthc Policy 2009;5: 68-86.

Affiliations: From The Centre for Health Services and Policy Research (Law, Cheng, Heard, Morgan), School of Population and Public Health, University of British Columbia, Vancouver, BC; the Department of Medicine (Dhalla), University of Toronto, Toronto, Ont.; the Li Ka Shing Knowledge Institute (Dhalla), St. Michael's Hospital, Toronto, Ont.; and the Institute for Clinical Evaluative Sciences (Dhalla), Toronto, Ont.

Contributors: Michael Law contributed to the conception and design of the study, acquired, analyzed and interpreted the data, and drafted the manuscript. Lucy Cheng contributed to the design of the study, analyzed and interpreted the data, and revised the manuscript for important intellectual content. Irfan Dhalla analyzed and interpreted the data and revised the manuscript for important intellectual content. Deborah Heard contributed to the design of the study, interpreted the data and revised the manuscript for important intellectual content. Steven G. Morgan contributed to the conception and design of the study, acquired, analyzed and interpreted the data, and revised the manuscript for important intellectual content. All of the authors approved the final version submitted for publication.

Acknowledgements: This analysis of Canadian Community Health Survey data on cost-related nonadherence was done under contract for the Office of Pharmaceuticals Management Strategies, Strategic Policy Branch, Health Canada. Michael Law received salary support through a New Investigator Award from the Canadian Institutes of Health Research, a Career Investigator Award from the Michael Smith Foundation for Health Research, and an Early Career Scholar Award from the Peter Wall Institute for Advanced Studies. Irfan Dhalla received salary support through a Fellowship Award from the Canadian Institutes of Health Research. 